\title{
THE BINDING ENERGY OF A $\Lambda$-PARTICLE IN NUCLEAR MATTER: A COMPARISON OF TWO FORMULATIONS*
}

\author{
R. F. BISHOP ** \\ Institute of Theoretical Physics, Department of Physics, \\ Stanford University, Stanford, California
}

Received 24 November 1969

\begin{abstract}
Two approaches to the problem of calculating the binding energy $B_{\Lambda}$ of a $\Lambda$-particle in nuclear matter are discussed. The first method is via the BetheGoldstone equation for the problem in the independent-pair approximation. The second method is a Green-function formulation which sums the ladder diagrams for the self-energy of the $\Lambda$-particle. Using an $S$-wave separable potential fitted to the $\Lambda \mathrm{N}$ scattering data, exact analytic expressions for $B \Lambda$ are found for both methods and compared. The relation between the two approaches is discussed and it is shown how to extend the Green-function formulation to include the effect of the higher-order cluster diagrams, contributing to the $\Lambda$-particle sclf-cnergy, in a consistent manner. It is pointed out that this approach provides a more systematic formulation than the usual extended Bethe-Goldstone approach. The model $\Lambda N$ hard core problem is also investigated in the Green function approach in an appendix: the ground-state energy is rederived and expressions are found for the effective mass and damping of the $\Lambda$-quasi-particle up through terms of order $\left(k_{\mathrm{F}} a\right)^{2}$.
\end{abstract}

\section{INTRODUCTION}

The purpose of this paper is to compare two methods for computing the binding energy for a $\Lambda$-particle in nuclear matter; where the $\Lambda$-particle thus plays the role of an impurity. Since it is distinct from the nucleons, it will be at rest in the ground state, as the exclusion principle applies only to the nucleons. Nuclear matter is considered to consist of equal numbers $\frac{1}{2} A$ of protons and neutrons interacting via purely nuclear forces, i.e. the electromagnetic interaction is assumed to be 'switched off'. The binding energy $B_{\Lambda}$ is defined by $-B_{\Lambda}=\lim _{A \rightarrow \infty}\left(E_{A+\Lambda}-E_{A}\right)$, where $E_{A}$ is the ground state energy of a self-bound quantity of nuclear matter, and $E_{A+\Lambda}$ is the

* Research sponsored in part by the Air Force Office of Scientific Research, Office of Aerospace Research, US Air Force, under AFOSR Contract No. F4462068-C-0075.

** Part of the work was completed whlle the author held a NATO Predoctoral Scholarship. 
energy of the self-bound system formed by adding a $\Lambda$-particle. Since the nuclear matter is assumed to be in equilibrium before the $\Lambda$-particle is added (i.e. $\partial E / \partial V=0$ ), we calculate $B_{\Lambda}$ by assuming the addition occurs at constant volume.

There are two possible reasons for considering this problem. The first is to test our theories of nuclear matter, by assuming that the $\Lambda \mathrm{N}$ interaction is known and comparing the theoretical value of $B_{\Lambda}$ with the experimental value [1-4]. Alternatively, we may consider that our theories of nuclear matter arc sufficicntly precise to use the known value of $B_{\Lambda}$ as a means of learning about the $\Lambda \mathrm{N}$ interaction. Whilst this second reason is propably the more valid, and provides the philosophy behind most recent work on the subject, in the present paper we take the first view.

Since the exact many-body problem is so complicated, one has to resort to approximate calculations. The philosophy behind this paper is that it is of interest to compare two such approaches.

The first method we shall consider is via the Bethe-Goldstone equation in the independent-pair approximation [1,5-7]. Essentially this method seeks to solve the two-particle Schrödinger equation in the presence of the rest of the nuclear matter, which is supposed to act as a background whose sole function is to restrict the states available to the interacting pair, through the exclusion principle. Henceforth we shall refer to this method as the BG method.

The other approach we consider is a Green-function method where the $\Lambda \mathrm{N}$ 'ladder diagrams' for the $\Lambda$-particle proper self energy, $\Sigma(p){ }^{*}$ are summed as Feynman diagrams, and the binding energy $B_{\Lambda}$ is found from solving [8] $-B_{\Lambda}=\Sigma\left(0,-B_{\Lambda}\right)$. This is the method pioneered by Galitskii [9] for singular (e.g. hard-core) interaction potentials, henceforth referred to as the G-method.

Our aim is thus, not to make thoroughly realistic calculations in either approach, but instead to use a model which is both tractable and close enough to the real world to enable us to make an meaningful comparison. To do this, the form chosen for the $\Lambda \mathrm{N}$ interaction potential is a spin- and isospin-independent, central, S-wave, separable (and hence non-local) potential. Such a choice enables us to find an exact analytic solution in both cases. To the knowledge of the author, the first exact solution to the Galitskii approach for nuclear matter is presented here within the scheme outlined above, but without assuming any small parameter in order to make a perturbation expansion.

Our philosophy is to do as good a job as possible using just two-body forces, and the potential is chosen so that we can make a direct comparison of the two methods. The G-approach treats the thermodynamics of the problem correctly and the binding energy calculated is really the chemical potential of a $\Lambda$-particle in nuclear matter to the same approximation to which the self energy is calculated. Thus an exact Green-function approach would include all the so-called rearrangement energy terms. We shall show

* The notation for four-vectors is $p=\left(\boldsymbol{p}, p_{O}\right)$, and our system of units is such that $\hbar=c=1$. 
explicitly in our model and quite generally that the BG method gives asymptotically the same value for $B_{\Lambda}$ as the G-approach as the density of nuclear matter approaches zero.

In sects. 2 and 3 the two methods are described and the equations for $B_{\Lambda}$ derived. The $\Lambda \mathrm{N}$ interaction potential used in the calculations is described in sect. 4 and the results of the calculation in sect. 5 . In sect. 6 and appen$\operatorname{dix} \mathrm{A}$ the relations between the two approaches are discussed, and in sect. 7 a procedure is given to extend the G-approach to calculate the higher-order cluster contributions to $B_{\Lambda}$. It is pointed out that this procedure, using Feynman diagrams, is much simpler to handle than the extended BG approach using Goldstone diagrams where the self-consistency requirements are difficult to build in. In outlining the procedure for calculating higherorder contributions we do not restrict ourselves to separable potentials. In appendix $B$, the full power of the $\mathrm{G}$-approach is demonstrated, where we consider a pure hard core $\Lambda \mathrm{N}$ interaction potential and derive results for the effective mass and damping of the $\Lambda$-quasi-particle.

\section{BETHE-GOLDSTONE (BG) METHOD}

It is assumed from the outset that only $\Lambda \mathrm{N}$ two-body forces are important. Further we make the independent-pair approximation [7], where each $\Lambda \mathrm{N}$ pair is treated independently and the rest of the nuclear matter is supposed not to affect the (virtual) scatterings of the pair, except to define the volume in momentum space in which final states are available (i.e. the exclusion principle is taken into account properly). Also the background of nuclear matter acts to define a momentum-dependent potential in which the nucleon of the scattering pair moves. This single-particle potential is taken into account crudely but in a self-consistent manner by the effective mass approximation. We assume that the $\Lambda$-particle acquires no effective mass in this manner, because the two dominant mechanisms which produce one in the pure nuclear matter case are absent for the $\Lambda$-particle, since no exclusion principle acts to produce any exchange integrals and any exchange forces are neglected. (In the physical situation exchange forces could occur through $\mathrm{K}$-meson exchange).

The two-particle Hamiltonian is

$$
\hat{H}=\hat{T}_{\Lambda}+\hat{T}_{\mathrm{N}}+\hat{W}_{\mathrm{N}}+\hat{V}_{\Lambda \mathrm{N}}=\hat{H}_{\mathrm{O}}+\hat{V}_{\Lambda \mathrm{N}},
$$

where $\hat{T}$ is the kinetic energy operator. $\hat{W}_{\mathrm{N}}=\hat{W}_{\mathrm{N}}\left(\hat{\boldsymbol{k}}_{\mathrm{N}}\right)$ is the momentumdependent potential acting on the nucleon. In the effective mass approximation

$$
\hat{T}_{\mathrm{N}}+\hat{W}_{\mathrm{N}}=W_{\mathrm{O}}+\frac{\hat{k}_{\mathrm{N}}^{2}}{2 m_{\mathrm{N}}^{*}} .
$$

Let $\psi$ represent the two-particle wave functions satisfying

$$
\hat{H} \psi=E \psi \text {. }
$$


The functions $\phi_{n}$ are the products of plane waves satisfying

$$
\hat{H}_{\mathrm{o}} \phi_{n}=E_{n} \phi_{n}
$$

Since the functions $\phi_{n}$ form a complete set, we can make the expansion

$$
|\psi\rangle=\sum_{n}\left|\phi_{n}\right\rangle\left\langle\phi_{n} \mid \psi\right\rangle
$$

From eqs. (2.1) and (2.2):

$$
\left\langle\phi_{n}\left|V_{\Lambda \mathrm{N}}\right| \psi\right\rangle=\left(E-E_{n}\right)\left\langle\phi_{n} \mid \psi\right\rangle
$$

and hence

$$
|\psi\rangle=\left|\phi_{\mathrm{o}}\right\rangle+\sum_{n \neq 0} \frac{\left|\phi_{n}\right\rangle\left\langle\phi_{n}\left|V_{\Lambda \mathrm{N}}\right| \psi\right\rangle}{\left(E-E_{\mathrm{o}}\right)-\left(E_{n}-E_{\mathrm{o}}\right)},
$$

where we normalize $\left\langle\phi_{0} \mid \psi\right\rangle=1$.

When we consider the Fermi-sea background which is assumed filled (zero-temperature limit) and form the properly antisymmetrized total wave function of all $A$ nucleons plus the $\Lambda$-particle, its effect is to exclude all states $\left|\phi_{n}\right\rangle$ from the sum in eq. (2.3) with nucleon momentum less than the Fermi momentum $k_{\mathrm{F}}$.

Since $V_{\Lambda \mathrm{N}}$ is a function only of the relative coordinate of the pair, and hence their total momentum is conserved, we can transform to the c.m. frame where

$$
\begin{array}{ll}
\boldsymbol{P}=\boldsymbol{k}_{\Lambda}+\boldsymbol{k}_{\mathrm{N}}, & \boldsymbol{R}=\frac{m_{\mathrm{N}}^{*} \boldsymbol{x}_{\mathrm{N}}+m_{\Lambda} \boldsymbol{x}_{\Lambda}}{m_{\Lambda}+m_{\mathrm{N}}^{*}}, \\
\boldsymbol{k}=\frac{m_{\mathrm{N}}^{*} \boldsymbol{k}_{\Lambda}-m_{\Lambda} \boldsymbol{k}_{\mathrm{N}}}{m_{\Lambda}+m_{\mathrm{N}}^{*}}, & \boldsymbol{x}=\boldsymbol{x}_{\Lambda}-\boldsymbol{x}_{\mathrm{N}},
\end{array}
$$

and writing

$$
\psi=\Omega^{-\frac{1}{2}} \exp (i \boldsymbol{P} \cdot \boldsymbol{R}) \psi(\boldsymbol{x}),
$$

where $\Omega$ is the normalization volume, we find

$$
\psi_{\boldsymbol{P}, \boldsymbol{K}}(\boldsymbol{x})=\mathrm{e}^{i \boldsymbol{k} \cdot \boldsymbol{x}}+\int \frac{\mathrm{d}^{3} t^{\prime}}{(2 \pi)^{3}} \theta\left(\left|\frac{\mu^{*}}{m_{\Lambda}} \boldsymbol{P}-\boldsymbol{t}^{\prime}\right|-k_{\mathrm{F}}\right) \mathrm{e}^{i \boldsymbol{t}^{\prime} \cdot \boldsymbol{x}\left\langle\frac{\left.\boldsymbol{t}^{\prime}|V| \psi\right\rangle}{\boldsymbol{K}^{2}-\boldsymbol{t}^{2}} 2 \mu^{*},\right.}
$$

where $\mu^{*}=m_{\Lambda} m_{\mathrm{N}}^{*} /\left(m_{\Lambda}+m_{\mathrm{N}}^{*}\right)$ is the reduced effective mass and the stepfunction 


$$
\begin{gathered}
\theta(x)= \begin{cases}1 & x>0 \\
0 & x<0\end{cases} \\
\boldsymbol{K}^{2}=\boldsymbol{k}^{2}+\frac{1}{\Omega} \int \mathrm{d}^{3} x \mathrm{e}^{-i \boldsymbol{k} \cdot \boldsymbol{x}} 2 \mu^{*} V(\boldsymbol{x}) \psi_{\boldsymbol{P}, \boldsymbol{k}}(\boldsymbol{x}) .
\end{gathered}
$$

From eq. (2.5), in the limit $\Omega \rightarrow \infty$ of a large volume of nuclear matter, $K^{2}-k^{2} \rightarrow 0$, since $V$ represents a force of finite range. Hence, since the denominator in eq. (2.4) never goes to zero except exactly at the Fermi surface, we replace $K^{2}$ by $k^{2}$. It is well-known that this is not a good approximation in the case of superconducting fermion systems. Since the energy gap in nuclear matter is known to be small [10], we may safely make the approximation in our discussion where we are concerned only with the binding energy.

Defining Fourier transforms $f(t)=\int \mathrm{e}^{-i \boldsymbol{t} \cdot \boldsymbol{x}} f(\boldsymbol{x}) \mathrm{d}^{3} x$ and writing $u=2 \mu^{*} V$, eq. (2.4) becomes

$$
\begin{array}{r}
\psi(t, k ; P)=(2 \pi)^{3} \delta(t-k)+\frac{\theta\left(\left|\frac{\mu^{*}}{m_{\Lambda}} P-t\right|-k_{F}\right)}{k^{2}-t^{2}+i \epsilon} \\
\quad \times \int \frac{\mathrm{d}^{3} t^{\prime}}{(2 \pi)^{3}}\left\langle t|u| t^{\prime}\right\rangle \psi\left(t^{\prime}, k ; P\right),
\end{array}
$$

where the positive infinitesimal $\epsilon$ is introduced as usual for scattered waves.

The energy shift per pair is now given by

$$
\Delta E(\boldsymbol{k}, \boldsymbol{P})=\frac{1}{\Omega} \int \frac{\mathrm{d}^{3} t}{(2 \pi)^{3}}\langle\boldsymbol{k}|\boldsymbol{V}| \boldsymbol{t}\rangle \psi(\boldsymbol{t}, \boldsymbol{k} ; \boldsymbol{P})
$$

and the total energy shift for the $\Lambda$-particle is

$$
-B_{\Lambda}\left(\boldsymbol{k}_{\Lambda}\right)=4 \Omega \int_{\frac{\mathrm{d}^{3} k_{\mathrm{N}}}{(2 \pi)^{3}}} \theta\left(k_{\mathrm{F}}-k_{\mathrm{N}}\right) \Delta E(\boldsymbol{k}, \boldsymbol{P}),
$$

where the factor 4 in eq. (2.8) accounts for the four spin and isospin degrees of freedom per nucleon.

If we now consider a separable potential of the form discussed in sect. 4

$$
\left\langle\boldsymbol{k}|V| \boldsymbol{k}^{*}\right\rangle=\frac{\hbar^{2}}{2 \mu} \lambda v(k) v\left(k^{\prime}\right) ; \quad \mu=\frac{m_{\Lambda} m_{\mathrm{N}}}{m_{\Lambda}{ }^{+m_{\mathrm{N}}}},
$$

eqs. (2.6)-(2.8) are readily solved in the case $\boldsymbol{k}_{\Lambda}=0$ to give

$$
\begin{aligned}
& -B_{\Lambda}^{(\mathrm{BG})}=\frac{4 \lambda^{*}}{2 \mu^{*}} \int \frac{\mathrm{d}^{3} k}{(2 \pi)^{3}} \theta\left(k_{\mathrm{F}}-k\right) v^{2}\left(\frac{\mu^{*}}{m_{\mathrm{N}}^{*}} k\right) \\
& \times\left[1+\lambda^{*} \int \frac{\mathrm{d}^{3} k^{\prime}}{(2 \pi)^{3}} \frac{v^{2}\left(k^{\prime}\right) \theta\left(\left|\frac{\mu^{*}}{m_{\Lambda}} k-k^{\prime}\right|-k_{\mathrm{F}}\right)}{\left\{k^{\prime 2}-\left(\frac{\mu^{*}}{m_{\mathrm{N}}^{*}} k\right)^{2}\right.}\right\}^{-1},
\end{aligned}
$$


where

$$
\lambda^{*}=\frac{\mu^{*}}{\mu} \lambda,
$$

for the binding energy $B_{\Lambda}(\mathrm{BG})=B_{\Lambda}(0)$ for a $\Lambda$-particle at rest in nuclear matter. It is important to note that this can now be evaluated exactly.

\section{GALITSKII (G) METHOD}

We define a $\Lambda$-particle Green-function propagator as

$$
G_{\Lambda}\left(x-x^{\prime}\right)_{\alpha \beta}=-i\left\langle A\left|T\left(\tilde{\Lambda}_{\alpha}(x) \tilde{\Lambda}_{\beta}^{\dagger}\left(x^{\prime}\right)\right)\right| A\right\rangle,
$$

where $\tilde{\Lambda}_{\alpha}(x)$ is the Heisenberg operator for the $\Lambda$-field, and $|A\rangle$ is the exact nuclear matter Heisenberg ground state. The nucleon propagator $G_{\mathrm{N}}$ is defined similarly in the usual way. The function $G_{\Lambda}$ defined above propagates only forward in time, and in terms of the usual proper self energy $\Sigma(p), G_{\Lambda}$ takes the form

$$
G_{\Lambda}(p)_{\alpha \beta}=\left[p_{\mathrm{o}}-\frac{p^{2}}{2 m_{\Lambda}}-\Sigma_{\alpha \beta}(p)+i \eta\right]^{-1} \mathrm{e}^{i p_{\mathrm{o}} \epsilon} \delta_{\alpha \beta},
$$

where $\eta, \epsilon$ are positive infinitesimals.

We now make the approximation that the only Feynman graphs we keep for $\Sigma$ are the ladder diagrams shown in fig. 1 . The expansion is made in terms of $G_{\Lambda}^{0}$, the unperturbed $\Lambda$-particle propagator, where $\Sigma_{\alpha \beta}\left(k_{\Lambda}\right)=0$, and in terms of the self-consistent nuclear-matter nucleon propagator $G_{\mathrm{N}}$. We assume that $G_{\mathrm{N}}$ is known from studies of nuclear matter and make the simplifying assumption as before that the nucleon single-particle energies are given by $\epsilon(k)=W_{\mathrm{O}}+\boldsymbol{k}^{2} / 2 m_{\mathrm{N}}^{*}$, giving $G_{\mathrm{N}}$ as

$$
G_{\mathrm{N}}(k)=\left[k_{\mathrm{O}}-W_{\mathrm{O}}-\frac{k^{2}}{2 m_{\mathrm{N}}^{*}}+i \eta \operatorname{sgn}\left(k-k_{\mathrm{F}}\right)\right]^{-1} \mathrm{e}^{i p_{\mathrm{O}} \epsilon}
$$

We now define a function $\Gamma$ which sums the $\Lambda \mathrm{N}$ ladders for multiple scatterings between the pair as shown in fig. 2a. This Born-Liouiville series for $\Gamma$ can be summed diagramatically as in fig. $2 \mathrm{~b}$ which corresponds to the integral equation

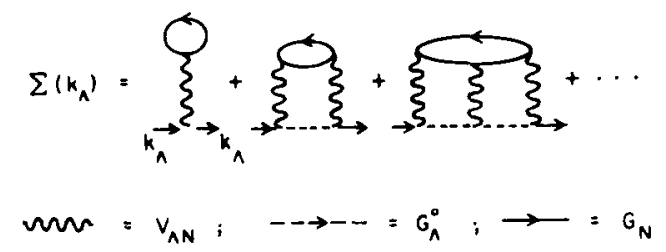

Fig. 1. The ladder diagrams for the $\Lambda$-particle proper self-energy. 


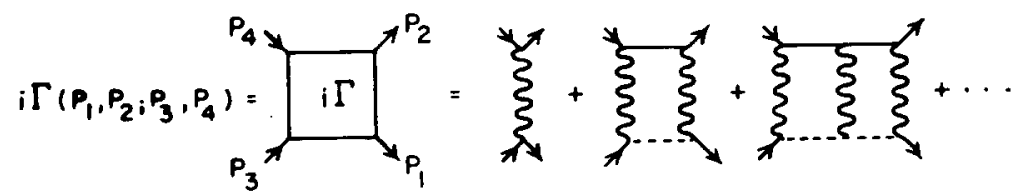

(a)
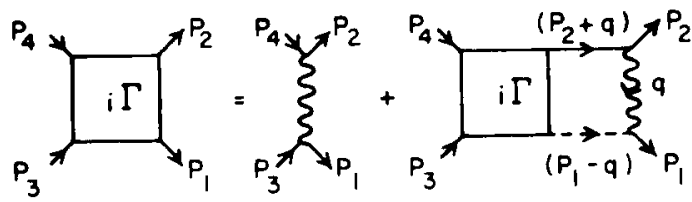

(b)

Fig. 2. (a) The $\Lambda \mathrm{N}$ scattering diagrams, (b) summed diagramatically as the BetheSalpeter equation.

$$
\begin{array}{r}
i \Gamma\left(p_{1}, p_{2} ; p_{3}, p_{4}\right)=i V\left(p_{1}, p_{2} ; p_{3}, p_{4}\right)-\int \frac{d^{4} q}{(2 \pi)^{4}} \Gamma\left(p_{1}-q, p_{2}+q ; p_{3}, p_{4}\right) \\
\times G_{N}\left(p_{2}+q\right) G_{\Lambda}^{\mathrm{O}}\left(p_{1}-q\right) V\left(\boldsymbol{p}_{1}-\boldsymbol{q}, \boldsymbol{p}_{2}+\boldsymbol{q} ; \boldsymbol{p}_{3}, \boldsymbol{p}_{4}\right),
\end{array}
$$

which is the Bethe-Salpeter equation for this problem. Since the total momentum of the pair is conserved, $\Gamma$ can be written as a function of three momentum differences. It is convenient to transform to the c.m. frame where

$$
\begin{gathered}
P=p_{1}+p_{2}=p_{3}+p_{4}, \quad p=\frac{1}{m_{\Lambda}+m_{\mathrm{N}}^{*}}\left(m_{\mathrm{N}}^{*} p_{1}-m_{\Lambda} p_{2}\right), \\
p^{\prime}=\frac{1}{m_{\Lambda}+m_{\mathrm{N}}^{*}}\left(m_{\mathrm{N}}^{*} p_{3}-m_{\Lambda} p_{4}\right), \quad \alpha=\frac{m_{\Lambda}-m_{\mathrm{N}}^{*}}{m_{\Lambda}+m_{\mathrm{N}}^{*}}, \\
\left\langle\boldsymbol{p}|V| \boldsymbol{p}^{\prime}\right\rangle=V\left(\boldsymbol{p}_{1}, \boldsymbol{p}_{2} ; \boldsymbol{p}_{3}, \boldsymbol{p}_{4}\right) .
\end{gathered}
$$

If we define a new function $x$, by

$$
\Gamma\left(\boldsymbol{p}, \boldsymbol{p}^{\prime} ; P\right) \equiv \Gamma\left(p_{1}, p_{2} ; p_{3}, p_{4}\right)=\int \frac{\mathrm{d}^{3} q}{(2 \pi)^{3}}\langle\boldsymbol{p}|V| \boldsymbol{q}\rangle \times\left(\boldsymbol{q}, \boldsymbol{p}^{\prime} ; P\right),
$$

then it is obvious from eq. (3.1) that $x$ satisfies the integral equation

$$
\begin{array}{r}
\chi\left(\boldsymbol{p}, \boldsymbol{p}^{\prime} ; P\right)=(2 \pi)^{3} \delta\left(\boldsymbol{p}^{\prime}-\boldsymbol{p}\right)+i \int \frac{\mathrm{d} p_{\mathrm{O}}}{2 \pi} G_{\Lambda}^{\mathrm{O}}\left(\frac{1}{2}(1+\alpha) P+p\right) G_{\mathrm{N}}\left(\frac{1}{2}(1-\alpha) P-p\right) \\
\quad \times \int \frac{\mathrm{d}^{3} q}{(2 \pi)^{3}}\langle\boldsymbol{p}|V| \boldsymbol{p}-\boldsymbol{q}\rangle \times\left(\boldsymbol{p}-\boldsymbol{q}, \boldsymbol{p}^{\prime} ; P\right),
\end{array}
$$

and that $\Gamma$ does not depend on $p_{\mathrm{O}}$ or $p_{\mathrm{O}}^{\prime}$ as written explicitly in eq. (3.2) 
Replacing the propagators in eq. (3.3) by their explicit forms, and defining

$$
E=P_{\mathrm{o}}-\frac{p^{2}}{2\left(m_{\Lambda}+m_{\mathrm{N}}^{*}\right)}-W_{\mathrm{o}}
$$

we find

$$
\begin{aligned}
\chi\left(\boldsymbol{p}, \boldsymbol{p}^{\prime} ; P\right)=(2 \pi)^{3} \delta\left(\boldsymbol{p}^{\prime}-\boldsymbol{p}\right)+\frac{\theta\left(\left|\frac{1}{2}(1-\alpha) \boldsymbol{P}-\boldsymbol{p}\right|-k_{\mathrm{F}}\right)}{\left(E-\frac{\boldsymbol{p}^{2}}{2 \mu^{*}}+i \eta\right)} \\
\quad \times \int \frac{\mathrm{d}^{3} q}{(2 \pi)^{3}}\langle\boldsymbol{p}|V| \boldsymbol{q}\rangle \times\left(\boldsymbol{q}, \boldsymbol{p}^{\prime} ; P\right),
\end{aligned}
$$

and hence, from eq. (3.1)

$$
\begin{array}{r}
2 \mu^{*} \Gamma\left(\boldsymbol{p}, \boldsymbol{p}^{\prime} ; P\right)=\left\langle\boldsymbol{p}|u| \boldsymbol{p}^{\prime}\right\rangle+\int \frac{\mathrm{d}^{3} k^{\prime}}{(2 \pi)^{3}}\left\langle\boldsymbol{p}|u| \boldsymbol{k}^{\prime}\right\rangle \frac{\theta\left(\left|\frac{1}{2}(1-\alpha) \boldsymbol{P}-\boldsymbol{k}^{\prime}\right|-k_{\mathrm{F}}\right)}{\epsilon-\boldsymbol{k}^{\prime 2}+i \eta} \\
\times 2 \mu^{*} \Gamma\left(\boldsymbol{k}^{\prime}, \boldsymbol{p}^{\prime} ; P\right),
\end{array}
$$

where

$$
\epsilon=2 \mu^{*} E ; \quad u=2 \mu^{*} V
$$

If we now assume a separable potential given by eq. (2.9), then eq. (3.6) can be solved explicitly to give

$$
2 \mu^{*} \Gamma\left(\boldsymbol{p}, \boldsymbol{p}^{\prime} ; P\right)=\lambda^{*} v(p) v\left(p^{\prime}\right) D^{-1}(P),
$$

where

$$
D(P)=1-\lambda^{*} \int \frac{\mathrm{d}^{3} k^{\prime}}{(2 \pi)^{3}} \frac{\theta\left(\left|\frac{1}{2}(1-\alpha) \boldsymbol{P}-\boldsymbol{k}^{\prime}\right|-k_{\mathrm{F}}\right)}{\epsilon-\boldsymbol{k}^{2}+i \eta} v^{2}\left(k^{\prime}\right) .
$$

Now, in terms of the function $\Gamma$, the self-energy $\Sigma$ is given by

$$
\begin{aligned}
\Sigma(p) & =-4 i \int \frac{\mathrm{d}^{4} k}{(2 \pi)^{4}} G_{\mathrm{N}}(k) \Gamma(p, k ; p, k) \\
& =-4 i \int \frac{\mathrm{d}^{4} k}{(2 \pi)^{4}} G_{\mathrm{N}}(k) \Gamma(\boldsymbol{q}, \boldsymbol{q} ; P),
\end{aligned}
$$

where

$$
\boldsymbol{q}=\frac{1}{m_{\Lambda}+m_{\mathrm{N}}^{*}}\left(m_{\mathrm{N}}^{*} \boldsymbol{p}-m_{\Lambda} k\right) ; \quad P=p+k .
$$

The $\Lambda$-quasi-particle energy spectrum is given by the poles of the propagator and according to Burkhardt $[8]$ we can identify $-B_{\Lambda}$ with the energy of a $\Lambda$-quasi-particle at rest $(\boldsymbol{p}=0)$. Thus, $B_{\Lambda}$ is given by the solution to 


$$
-B_{\Lambda}=\Sigma\left(0,-B_{\Lambda}\right)
$$

If we now put in the explicit form for $G_{\mathrm{N}}(k)$, and observe that considered as a function of $k_{\mathrm{O}}, D(P)$ is analytic in the upper half-plane when $\boldsymbol{p}=0$, we find

$$
\begin{aligned}
-B_{\Lambda}^{(\mathrm{G})}=\frac{4 \lambda^{*}}{2 \mu^{*}} \int \frac{\mathrm{d}^{3} k}{(2 \pi)^{3}} & \theta\left(k_{\mathrm{F}}-k\right) v^{2}\left(\frac{\mu^{*}}{m_{\mathrm{N}}^{*}} k\right) \\
\times & {\left.\left[1+\lambda^{*} \int \frac{\mathrm{d}^{3} k^{\prime}}{(2 \pi)^{3}} \frac{v^{2}\left(k^{\prime}\right) \theta\left(\left|\frac{\mu^{*}}{m_{\Lambda}}-\boldsymbol{k}^{\prime}-\boldsymbol{k}^{\prime}\right|-k_{\mathrm{F}}\right)}{-\mu^{*}} \boldsymbol{k}_{\mathrm{N}}^{*}\right)^{2}+2 \mu^{*} B_{\Lambda}^{(\mathrm{G})}\right]^{-1}, }
\end{aligned}
$$

which is an implicit equation for $B_{\Lambda}^{(\mathrm{G})}$, and should be compared with eq. (2.10) for $B_{\Lambda}^{(B G)}$.

\section{THE $\Lambda$ N SEPARABLE INTERACTION}

The real $\Lambda \mathrm{N}$ interaction is presumably approximately local, of short range, and includes a hard core of about the same radius as the NN interaction [11]. It almost certainly contains non-central forces [4] and is slightly spin dependent [12]. The latest evidence [12] indicates that its strength may be reduced in odd angular momentum partial waves.

We have seen that it is very convenient to use a separable potential however, in order to obtain an exact solution for the binding energy. We shall use a one-term, $\mathrm{S}$-wave, separable potential which is capable of reproducing the effects of the hard core in the range of energies with which we are concerned. Such a potential is, of course, very oversimplified but contains the most important aspects of the real interaction. We insist that the potential fit such low energy $\Lambda \mathrm{N}$ scattering data as is available $[13,14]$, including the scattering length and effective range.

In momentum space the $S$ - wave interaction is given by eq. (2.9) where $\boldsymbol{k}$ and $\boldsymbol{k}^{\prime}$ are the initial and final relative momenta. It is readily shown that the $S$-wave phase shift $\delta_{\mathrm{O}}(k)$ is then given by

$$
k \operatorname{ctg} \delta_{\mathrm{O}}(k)=-4 \pi \frac{\left[1+\frac{\lambda}{2 \pi^{2}} G(k)\right]}{\lambda v^{2}(k)} \approx-\frac{1}{a_{\mathrm{O}}}+\frac{1}{2} r_{\mathrm{O}} k^{2},
$$

where $G(k)$ is the principal value (P.V.) integral

$$
G(k)=\mathrm{P} . \mathrm{V} \cdot \int_{0}^{\infty} \mathrm{d} q \frac{q^{2} v^{2}(q)}{q^{2}-k^{2}},
$$


and where eq. (4.1) also defines the low-energy scattering parameters $a_{\mathrm{o}}$ and $r_{\mathrm{O}}$.

We now use an idea of Tabakin [15] to allow for both attraction and repulsion in the one-term potential. The basic idea is that the phase shift $\delta_{0}$ given by eq. (4.1) will not in general change sign (which must occur for a realistic potential which includes a hard core and an attractive tail) without passing through $\frac{1}{2} \pi$, which is not allowed for an unbound system such as $\Lambda \mathrm{N}$. However, we can arrange for the numerator of eq. (4.1) to vanish at some momentum $k_{\mathrm{c}}$ if we impose also that $v\left(k_{\mathrm{c}}\right)=0$, and the ratio

$$
\frac{\left[1+\frac{\lambda}{2 \pi^{2}} G\left(k_{\mathrm{c}}\right)\right]}{v^{2}\left(k_{\mathrm{c}}\right)}=0 .
$$

Thus, if we can choose a function $v(k)$ which satisfies both of these conditions, we may avoid the phase shift of $\frac{1}{2} \pi$ and produce instead the sign change expected of a potential with short-range repulsion and an attractive tail.

Using a simplified form of a suggestion by Tabakin [15] we choose

$$
v(k)=\left(k_{c}^{2}-k^{2}\right) /\left(k^{4}+a^{4}\right) \text {. }
$$

The values of $a, k_{\mathrm{c}}$ and $\lambda$ are fixed by using the condition given by eq. (4.3) and fitting to the low-energy scattering parameters $a_{\mathrm{O}}$ and $r_{\mathrm{O}}$. The experimental data is such that the parameters $a_{0}$ and $r_{0}$ cannot be evaluated unambiguously. We use the values of Herndon and Tang [12] who assume a hard core in the $\Lambda \mathrm{N}$ potential in the reduction of their data. The measured scattering amplitude can be written in terms of singlet and triplet components, $f^{1}$ and $f^{3}$ respectively, as

$$
f=\frac{1}{4}\left(f^{1}+3 f^{3}\right)+\frac{1}{4} \sigma_{\Lambda} \cdot \sigma_{\mathrm{N}}\left(f^{3}-f^{1}\right) .
$$

In a spin-saturated system as nuclear matter, the second term is zero. Since in the analysis of Herndon and Tang the parameters for singlet and triplet states are nearly the same, and since the triplet values (which are seen to be weighted three times as heavily as the singlet values) are better known than the singlet, we use their triplet values

$$
a_{\mathrm{o}}=-2.25 \mathrm{fm} ; \quad r_{\mathrm{o}}=3.40 \mathrm{fm} \text {. }
$$

For our choice of potential, $G(k)$ can be evaluated analytically as

$$
\begin{aligned}
G(k)=-\frac{\pi}{8 \sqrt{2}} \frac{1}{a^{5}\left(k^{4}+a^{4}\right)^{2}} & {\left[\left(a^{4}+k^{2} k_{c}^{2}\right)\left\{\left(k^{4}-a^{4}\right)\left(k_{\mathrm{c}}^{2}+a^{2}\right)-2 k^{2} a^{2}\left(k_{\mathrm{c}}^{2}-a^{2}\right)\right\}\right.} \\
+ & \left.3 a^{2}\left(k_{\mathrm{c}}^{2}-k^{2}\right)\left\{\left(k^{4}-a^{4}\right)\left(k_{\mathrm{c}}^{2}-a^{2}\right)+2 k^{2} a^{2}\left(k_{\mathrm{c}}^{2}+a^{2}\right)\right\}\right] .
\end{aligned}
$$




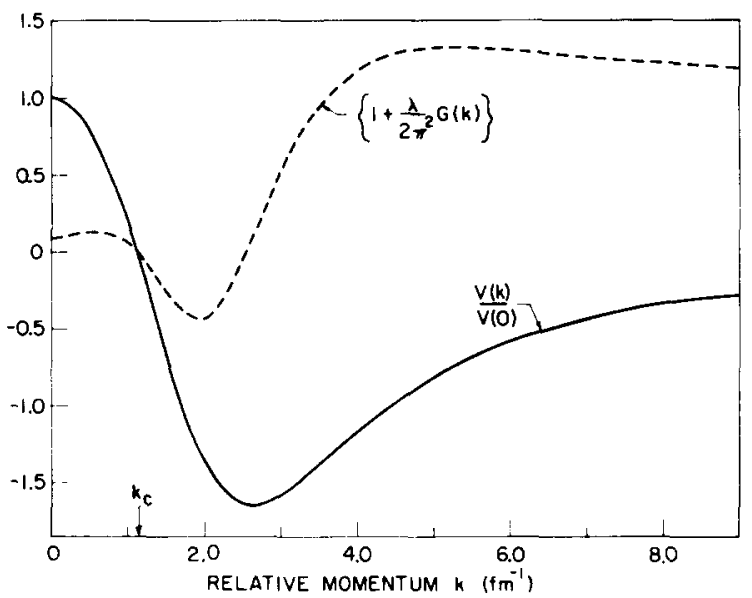

Fig. 3. The solid line represents the function $v(k)$ used in the separable potential eq. (2.9); and the dashed line represents the expression $\left\{1+\left(\lambda / 2 \pi^{2}\right) G(k)\right\}$ defined by eqs. (4.1) and (4.2).

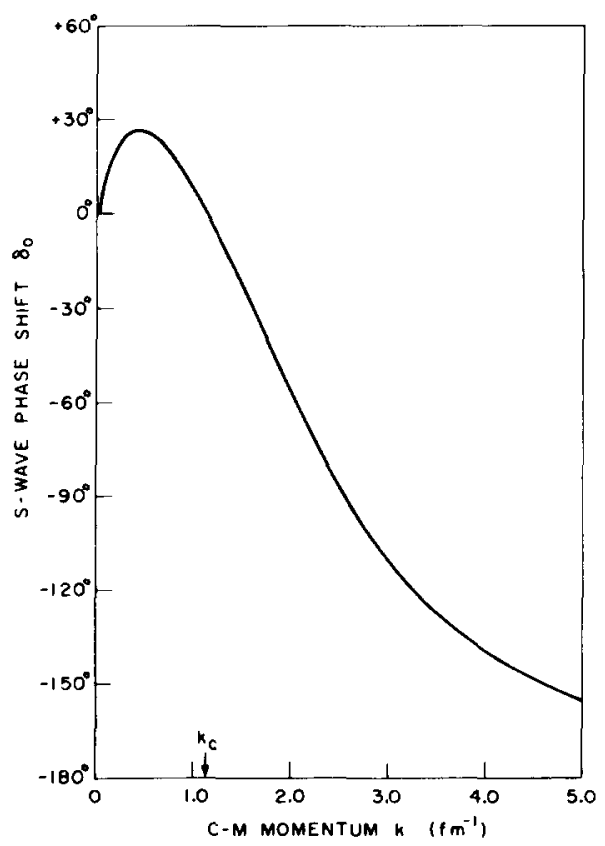

Fig. 4. The $\mathrm{S}$-wave phase shift $\delta_{\mathrm{O}}(k)$ produced by the separable potential as a function of the c.m. momentum $k$. 


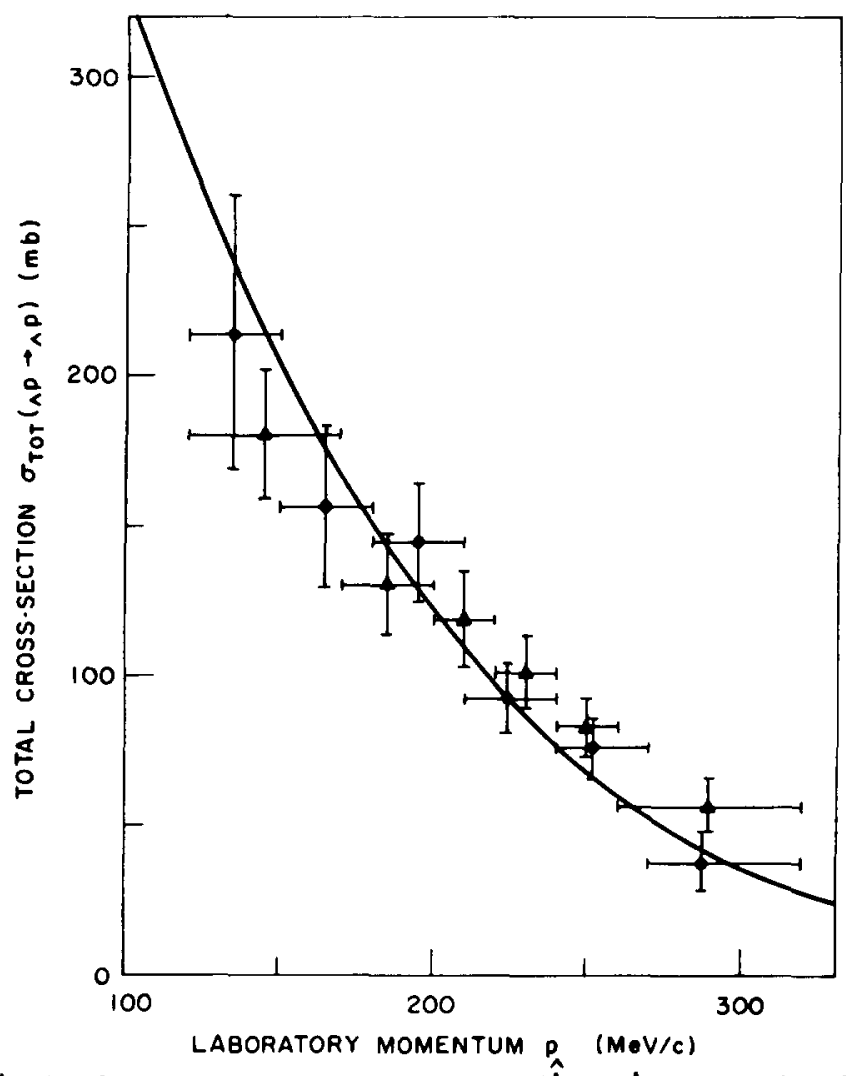

Fig. 5. The $\Lambda \mathrm{N}$ total scattering cross section $\sigma_{\text {tot }}\left(\hat{\Lambda}_{\mathrm{p}} \rightarrow \Lambda \mathrm{p}\right)$ assuming the nucleon in itially at rest and the $\Lambda$-particle of momentum $p_{\Lambda}$ in the lab frame. The solid curve is the theoretical prediction using the separable potential; the experimental points are from Alexander et al.: - ref. [13] and $\boldsymbol{\Delta}$ ref. [14].

The condition expressed by eq. (4.3) implies

and we find

$$
\lambda=\frac{-16 \pi \sqrt{2} a^{5}}{a^{2}-k_{\mathrm{c}}^{2}}
$$

$$
-\frac{1}{a_{\mathrm{o}}}=\frac{a\left(a^{2}-3 k_{\mathrm{c}}^{2}\right)}{4 \sqrt{2} k_{\mathrm{c}}^{2}}, \quad r_{\mathrm{O}}=\frac{\left(5 k_{\mathrm{c}}^{4}+a^{4}\right)}{2 \sqrt{2} a k_{\mathrm{c}}^{4}}
$$

These three equations have solution

$$
a=2.328 \mathrm{fm}^{-1}, \quad k_{\mathrm{c}}=1.140 \mathrm{fm}^{-1}, \quad \lambda=-1180 \mathrm{fm}^{-3} .
$$

In fig. (3) we plot $v(k)$ and the expression $\left[1+\left(\lambda / 2 \pi^{2}\right) G(k)\right]$, and in fig. 4 the phase shift $\delta_{0}(k)$, which has the form expected. 
As an independent check, we plot in fig. 5 the total cross section in the lab frame where the nucleon is at rest. It is seen to agree well with the experimental points, taken from Alexander et al. $[13,14]$.

\section{RESULTS}

The following points should be noted, concerning eqs. (2.10) and (3.10) for the binding energy $B_{A}$, by the two methods:

(i) It is apparent that the BG method is basically the low-density $\left(k_{\mathrm{F}} \rightarrow 0\right)$ limit of the $\mathrm{G}-$ method. This point is illustrated by fig. 6 .

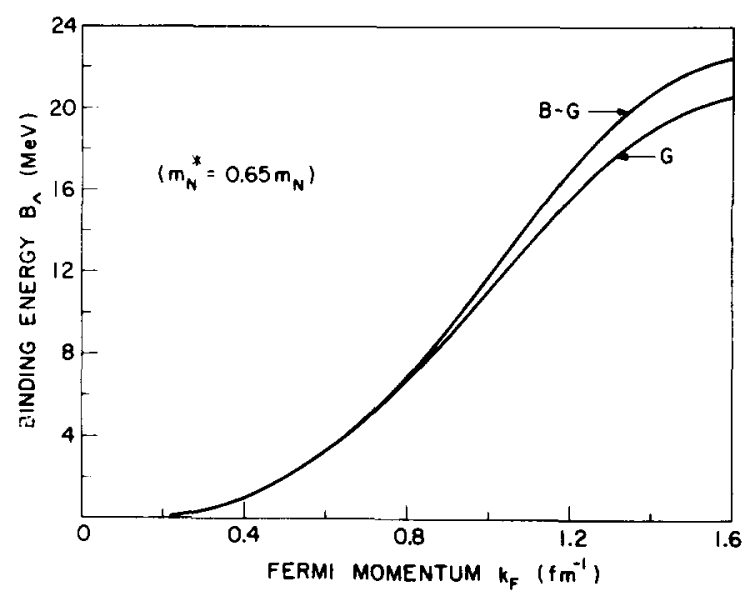

Fig. 6. The binding energy $B_{\Lambda}$ as a function of the Fermi momentum $k_{\mathrm{F}}$ at a nucleon effective mass $m_{\mathrm{N}}^{*}=0.65 m_{\mathrm{N}}$. The curve $\mathrm{G}$ is from the Galitskii method; the curve BG from the Bethe-Goldstone method.

(ii) Further, the BG result is a small parameter approximation to the G-result, in the sense that when either $m_{\mathrm{N}}^{*} / m_{\mathrm{N}}$ or the strength of the potential $(\lambda)$ goes to zero, the two methods agree. See also fig. (7) in relation to the first of these assertions.

(iii) The solution of the G-method always gives less binding than the solution of the BG method.

(iv) For sufficiently negative values of $\lambda$ (attractive tail) it would appear that the factor in square brackets in eqs. (2.10) and (3.10) can go to zero. This situation is closely related to the phenomenon of Cooper pairing, and should it arise, would be an indication of the insufficiency of the approximations made. In fact this factor was always positive in these calculations.

(v) If $\lambda$ is treated as a small parameter, and $B_{\Lambda}$ is expanded in powers of $\lambda$, the two methods always agree through second-order terms. In appendix $B$ we show that this is the case also for a local hard core potential, and in sect. 6 that this is a general property of the two solutions for all (not just separable) potentials. 


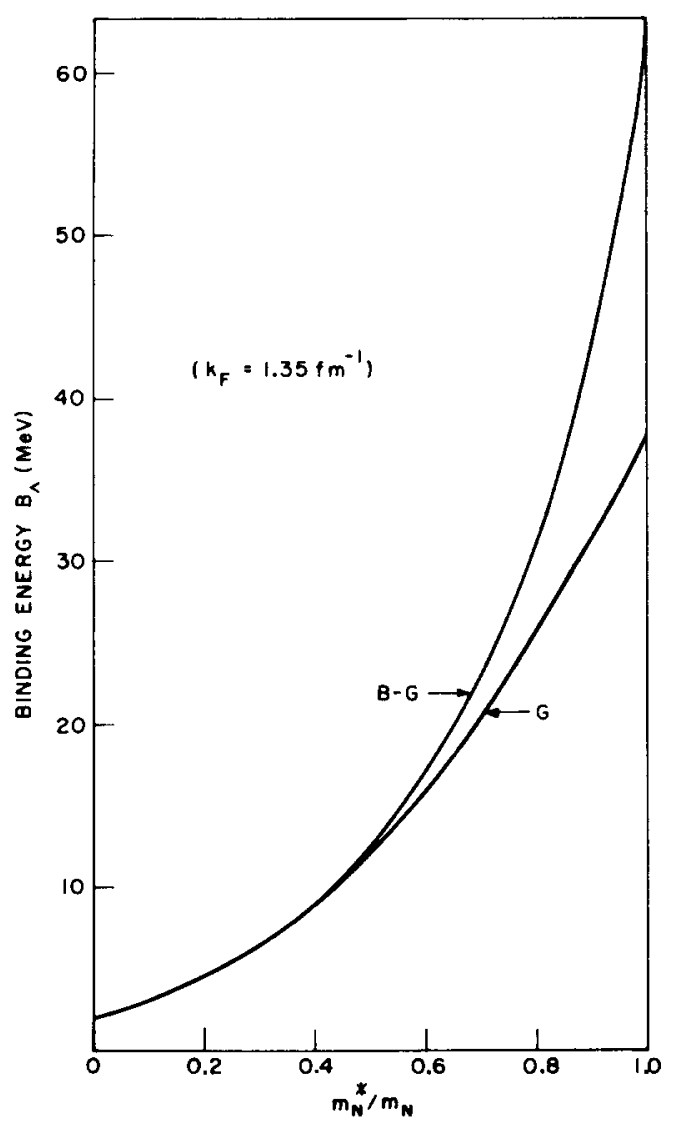

Fig. 7. The binding energy $B \Lambda$ as a function of the nucleon effective mass $m_{N}^{*}$ evaluated at a Fermi momentum $k_{\mathrm{F}}=1.35 \mathrm{fm}^{-1}$. The curve $\mathrm{G}$ is from the Galitskii method; the curve BG from the Bethe-Goldstone method.

After the trivial conversion of eqs. (2.10) and (3.10) to double integrals, the potential described in the last section was inserted, and the integrals performed numerically on a computer. A reasonable value for the nucleon effective mass in nuclear matter at the Fermi surface is $m_{\mathrm{N}}^{*}=0.65 m_{\mathrm{N}}$, as shown in ref. [5]. Using this value for $m_{\mathrm{N}}^{*}$, the values for $B_{\Lambda}$ as a function of $k_{\mathrm{F}}$ are shown in fig. (6) for the two methods. At the equilibrium density of nuclear matter, which corresponds to $k_{\mathrm{F}}=1.35 \mathrm{fm}^{-1}$, we find $B_{\Lambda}^{(\mathrm{BG})}=19.99 \mathrm{MeV}$ and $B_{\Lambda}^{(\mathrm{G})}=18.28 \mathrm{MeV}$.

In fig. $7, B_{\Lambda}$ is plotted as a function of $m_{\mathrm{N}}^{*}$ for $k_{\mathrm{F}}=1.35 \mathrm{fm}^{-1}$. At $m_{\mathrm{N}}^{*}=m_{\mathrm{N}}$ the $\mathrm{BG}$ and $\mathrm{G}-$ methods are in considerable discord, giving 62.6 and $37.5 \mathrm{MeV}$ respectively for the binding energy, whilst at $m_{\mathrm{N}}^{*}=0.65 m_{\mathrm{N}}$ the relative discrepancy is much smaller and of the order of $10 \%$. 


\section{COMPARISON OF THE G AND BG METHODS}

We now investigate exactly what we have achieved by using the G-method rather than the BG method. To this end we shall set up an integral equation connecting quantities defined in the two approaches. To make the analogy clearer let us write $\tilde{x}\left(p, p^{\prime} ; P\right)$ in place of the Bethe-Goldstone wave function $\psi\left(\boldsymbol{p}, \boldsymbol{p}^{\prime} ; \boldsymbol{P}\right)$ defined in sect. 2 , and define

$$
\widetilde{\Gamma}\left(\boldsymbol{p}, \boldsymbol{p}^{\prime} ; \boldsymbol{P}\right)=\int \frac{\mathrm{d}^{3} q}{(2 \pi)^{3}}\langle\boldsymbol{p}|V| \boldsymbol{q}\rangle \tilde{\chi}\left(\boldsymbol{q}, \boldsymbol{p}^{\prime} ; \boldsymbol{P}\right)
$$

Then we may rewrite eq. (2.6) as

$$
\begin{array}{r}
\tilde{\mathrm{x}}\left(\boldsymbol{p}, \boldsymbol{p}^{\prime} ; \boldsymbol{P}\right)-\frac{\theta\left(\left|\frac{1}{2}(1-\alpha) \boldsymbol{P}-\boldsymbol{p}\right|-k_{\mathrm{F}}\right)}{\epsilon-\boldsymbol{p}^{2}+i \eta} \int \frac{\mathrm{d}^{3} q}{(2 \pi)^{3}}\langle\boldsymbol{p}|u| \boldsymbol{q}\rangle \tilde{\mathrm{x}}\left(\boldsymbol{q}, \boldsymbol{p}^{\prime} ; \boldsymbol{P}\right) \\
=(2 \pi)^{3} \delta\left(\boldsymbol{P}-\boldsymbol{p}^{\prime}\right)+b\left(\boldsymbol{P}, \boldsymbol{p}^{\prime} ; \boldsymbol{P}\right),
\end{array}
$$

with

$b\left(\boldsymbol{p}, \boldsymbol{p}^{\prime} ; \boldsymbol{P}\right)=\theta\left(\left|\frac{1}{2}(1-\alpha) \boldsymbol{P}-\boldsymbol{p}\right|-k_{\mathrm{F}}\right) 2 \mu^{*} \widetilde{\boldsymbol{\Gamma}}\left(\boldsymbol{p}, \boldsymbol{p}^{\prime} ; \boldsymbol{P}\right)\left[\frac{1}{\boldsymbol{p}^{2}-\boldsymbol{p}^{2}+i \eta}-\frac{1}{\epsilon-\boldsymbol{p}^{2}+i \eta}\right]$.

The reason for writing eq. (6.1) in this manner, is that by observing eq. (3.5) we see that $x\left(p, p^{\prime} ; P\right)$ is the Green function for the left-hand side of eq. (6.1). Using the symmetry property $\Gamma\left(\boldsymbol{p}, \boldsymbol{p}^{\prime} ; P\right)=\Gamma\left(\boldsymbol{p}^{\prime}, \boldsymbol{p} ; P\right)$; it is readily proved that

$$
\widetilde{\chi}\left(\boldsymbol{p}, \boldsymbol{p}^{\prime} ; \boldsymbol{P}\right)=\chi\left(\boldsymbol{p}, \boldsymbol{p}^{\prime} ; P\right)+\int \frac{\mathrm{d}^{3} k}{(2 \pi)^{3}} \times(\boldsymbol{p}, \boldsymbol{k} ; P) b\left(\boldsymbol{k}, \boldsymbol{p}^{\prime} ; \boldsymbol{P}\right)
$$

and hence that

$$
\begin{aligned}
\Gamma\left(\boldsymbol{p}, \boldsymbol{p}^{\prime} ; P\right)=\widetilde{\Gamma}\left(\boldsymbol{p}, \boldsymbol{p}^{\prime} ; \boldsymbol{P}\right)+\int \frac{\mathrm{d}^{3} k^{\prime}}{(2 \pi)^{3}} \Gamma\left(\boldsymbol{p}, \boldsymbol{k}^{\prime} ; P\right) \theta\left(\left|\frac{1}{2}(1-\alpha) \boldsymbol{P}-\boldsymbol{k}^{\prime}\right|-k_{\mathrm{F}}\right) \\
\times\left[\frac{1}{\epsilon-\boldsymbol{k}^{2}+i \eta}-\frac{1}{\boldsymbol{p}^{\prime 2}-\boldsymbol{k}^{2}+i \eta}\right] 2 \mu^{*} \widetilde{\Gamma}\left(\boldsymbol{k}^{\prime}, \boldsymbol{p}^{\prime} ; \boldsymbol{P}\right) .
\end{aligned}
$$

Using eqs. (2.8), (3.8) and (6.3) and that $\Gamma\left(p, k^{\prime} ; P\right)$ is analytic in the upperhalf $k_{\mathrm{o}}^{\prime}$-plane for $\boldsymbol{p}=0$, we find

$$
\begin{aligned}
& \Sigma\left(O, p_{\mathrm{O}}\right)=-B_{\mathrm{V}}^{(\mathrm{BG})}+4 \int \frac{\mathrm{d}^{3} k}{(2 \pi)^{3}} \int \frac{\mathrm{d}^{3} k^{\prime}}{(2 \pi)^{3}} \theta\left(\left|\frac{1}{2}(1-\alpha) \boldsymbol{k}^{-} \boldsymbol{k}^{\prime}\right|-k_{\mathrm{F}}\right) \theta\left(k_{\mathrm{F}}-k\right) \\
& \times 2 \mu^{*} \widetilde{\Gamma}\left(\boldsymbol{k}^{\prime}, p^{\prime} ; P_{(0)}\right) \Gamma\left(q, \boldsymbol{k}^{\prime} ; P_{(0)}\right)\left[\frac{1}{2 \mu^{*} p_{\mathrm{O}}+\boldsymbol{q}^{2}-\boldsymbol{k}^{2}+i \eta}-\frac{1}{\boldsymbol{q}^{2}-\boldsymbol{k}^{\prime 2}+i \eta}\right],
\end{aligned}
$$

where 


$$
\boldsymbol{q}=-\frac{\mu^{*}}{m_{\mathrm{N}}^{*}} \boldsymbol{k}, \quad P(0)=\left(\boldsymbol{k}, p_{\mathrm{O}}+W_{\mathrm{O}}+\frac{\boldsymbol{k}^{2}}{2 m_{\mathrm{N}}^{*}}\right) \text {. }
$$

Hence, since $-B_{\Lambda}^{(\mathrm{G})}$ is the solution to $p_{\mathrm{O}}=\Sigma\left(0, p_{\mathrm{O}}\right)$, we have

$$
\begin{aligned}
& B_{\Lambda}^{(\mathrm{G})}=B_{\Lambda}^{(\mathrm{BG})}-4 B_{\Lambda}^{(\mathrm{G})} \int \frac{\mathrm{d}^{3} k}{(2 \pi)^{3}} \int \frac{\mathrm{d}^{3} k^{\prime}}{(2 \pi)^{3}} \theta\left(\left|\frac{\mu^{*}}{m_{\Lambda}} \boldsymbol{k}^{-} \boldsymbol{k}^{\prime}\right|-k_{\mathrm{F}}\right) \theta\left(k_{\mathrm{F}}-k\right) \\
& \times 2 \mu^{*} \tilde{\Gamma}\left(\boldsymbol{q}, \boldsymbol{k}^{\prime} ; \boldsymbol{P}_{(0)}\right) 2 \mu^{*} \Gamma\left(\boldsymbol{k}^{\prime}, \boldsymbol{q} ; P_{(0)}\right) \frac{1}{\left(\boldsymbol{k}^{\mathbf{2}^{2}}-\boldsymbol{q}^{2}\right)\left(\boldsymbol{k}^{\prime 2}-\boldsymbol{q}^{2}+2 \mu^{*} B_{\Lambda}^{(\mathrm{G})}\right)} .
\end{aligned}
$$

In the case of a separable $\Lambda \mathrm{N}$ potential the solutions obtained for $\Gamma$ and $\tilde{\Gamma}$ are readily shown to satisfy this equation, which is however valid for all potentials.

The first thing to notice from eq. (6.4) is that if $\lambda$ is a parameter characterising the strength of the potential, then $B(\mathrm{G})$ and $B(B G)$ agree to second order in $\lambda$ as previously asserted. Also the two methods obviously converge in the limit $k_{\mathrm{F}} \rightarrow 0$, where $\left(B^{(\mathrm{G})}-B^{(\mathrm{BG})}\right) / B(\mathrm{G}) \rightarrow 0$.

To compare the methods further let us look at the problem that we have tried to solve in three separate stages.

(a) First consider the hypothetical problem of a $\Lambda$-particle placed in a free Fermi gas of nucleons, i.e. nuclear matter with the NN interactions turned off. In both methods this is equivalent to putting $W_{\mathrm{O}}=0, m_{\mathrm{N}}^{*}=m_{\mathrm{N}}$. As we have seen, in this case the two methods give different results. As is well known the BG method also sums the same set of ladder graphs, but it assumes that the starting initial frequency of the $\Lambda$-particle is known and takes just its on-the-energy-shell value. In the G-method it is realized that what is being calculated is a self-energy insertion, and that the frequency of propagation of the $\Lambda$-particle in the medium is unknown until the problem is solved.

(b) Let us now turn on the NN interactions. In both cases the single-particle nucleon energies change. The methods still disagree but in no way essentially different from case (a). All that happens is that $m_{\mathrm{N}}$ gets replaced by $m_{\mathrm{N}}^{*}$. This has the consequence of making the relative difference between the two methods smaller. The reason for this is that lowering the mass of the nucleon makes it more difficult to excite intermediate states with the same momentum.

(c) Let us now carry our calculations one step further and make the BG method 'self-consistent' for the $\Lambda$-particle. This means that just as we assumed the nucleon of the $\Lambda \mathrm{N}$ pair considered moved in a single-particle potential $W_{N}\left(k_{N}\right)$, so does the $\Lambda$-particle. To formulate this self-consistency condition in general is quite difficult. However, to the extent that the $\Lambda$ acquires no effective mass (as always assumed) the sole effect is then to shift the $\Lambda$-particle energy spectrum by a constant amount. Since only energy differences arise in eq. (2.3) this has no effect on the solution at all. We might now be tempted to do the same thing for the G-method. That is, in the ladder diagrams of fig. (1) the unperturbed $\Lambda$-particle propagator would 
be replaced by the self-consistent propagator $G_{\Lambda}=\left[p_{0}-p^{2} / 2 m_{\Lambda}-V_{\Lambda}+\dot{m}\right]^{-1}$ and to be self-consistent we replace $V_{\Lambda}$ by $-B_{\Lambda}$ at the end of the calculation. The effect of this is to make the G and BG methods identical in their results. This might lead us to suspect that the BG method is correct and the G-method wrong. However the effect on the G-method of replacing the unperturbed intermediate $\Lambda$-propagator by the self-consistent propagator is effectively to sum a selected class of higher-order graphs (i.e. ones having two or more nucleon-hole lines). Insofar as we have neglected many other higher-order graphs this is erroneous, and we are forced to conclude that the G-method as originally presented is correct to the order (only graphs with one nucleon hole line) considered. A further discussion of this point, based on eq. (6.4) is relegated to appendix $A$.

\section{EXTENSION TO HIGHER ORDERS}

Both the BG and G-methods we have presented sum the ladder diagrams for the self-energy of the $\Lambda$-particle (all diagrams with one nucleon-hole line); the former treating them as Goldstone diagrams, the latter as Feynman diagrams. One might expect that the series expansion for $B_{\Lambda}$, of which we have found the lowest-order term, is one in the number of $\Gamma$-matrix interactions. Bethe [16] has shown however that such an expansion does not have good convergence properties for the nuclear-matter problem. He shows that the expansion is essentially one in powers of the density $\rho$, and the $n$th order diagrams for the self-energy are essentially all those containing $n$ nucleon hole lines, in close correspondence with the cluster expansion familiar in statistical mechanics. The expansion parameter in the nuclear-matter case is roughly the ratio of the volume of the hard core of the NN interaction to the volume per nucleon or some equivalent correlation parameter $\omega_{\mathrm{NN}}+$. In the case of calculating $B_{\Lambda}$ we expect similarly that the $n$th order contribution will come from summing all diagrams with $n$ nucleon-hole lines, and we shall henceforth adopt this standpoint. The second-order contribution to $B_{\Lambda}$ presumably depends on both correlation parameters $\omega_{\mathrm{NN}}$ and $\omega_{\Lambda \mathrm{N}}$ or some equivalent parameters.

From the discussion in appendix $A$, we see that our lowest- (first-) order calculations for $B(G)$ and $B(B G)$ that we have presented differ essentially by higher-order terms, and insofar as we have neglected such terms, the two (lowest-order) results must be considered identical. Their relative

$\neq$ In the Goldstone diagram approach the correlation parameter $\omega_{\mathrm{NN}}$ turns out to be $\omega_{\mathrm{NN}}=\rho \int\left\langle u_{\mathrm{NN}}^{2}\right\rangle \mathrm{d}^{3} r$, where $u_{\mathrm{NN}}$, the NN deficit wave function, defined as

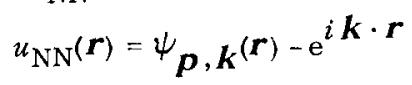

is the difference between the BG wave function for an NN pair and the free wave function of the uncorrelated pair. The averaging procedure is normally carried out by setting $\boldsymbol{P}=\boldsymbol{O}$ and averaging the $\boldsymbol{k}$-dependence over the filled Fermi sphere. The quantity $\omega_{\Lambda N}$ is defined in an analogous fashion. 
difference, however, gives a good indication of whether the cluster expansion is expected to converge well or not. In the case where $m_{\mathrm{N}}^{*}$ takes its physical value, their relative difference is about $10 \%$ for our model calculation.

Let us now consider how to extend our results to make a consistent second-order calculation for $B_{\Lambda}$. It is now that the power of the Green-function method becomes apparent. The first-order proper self-energy $\Sigma_{1}$ is first calculated as we have already done from the diagrams of fig. (1), where the intermediate $\Lambda$-particle propagator is $G_{\Lambda}^{0}$ so that we do not overcount when we look at diagrams for $\Sigma_{2}$. The second-order proper self-energy $\Sigma_{2}$ is then calculated from the diagrams of figs. $8 a$ and $8 b$, where the sawtooth lines represent a $\Gamma_{\Lambda \mathrm{N}}$ or $\Gamma_{\mathrm{NN}}$ matrix interaction, as shown in fig. $8 \mathrm{c}$, for ease of drawing.

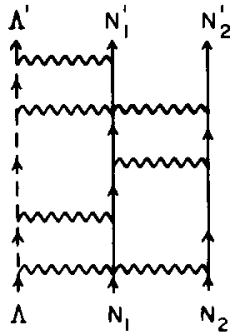

(a)

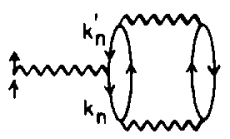

(b)

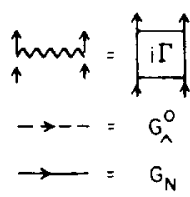

(c)

Fig. 8. All diagrams containing two nucleon-hole lines which contribute to the $\Lambda$-particle proper self-energy, as explained in the text.

The diagrams, of which fig. $8 \mathrm{a}$ is representative represent all diagrams formed by drawing a $\Lambda$-particle line and two nucleon lines all with arrows in the same direction, connected by any number of $N N$ and $\Lambda N \Gamma$-matrix interactions (excluding diagrams in which there is more than one consecutive $\Gamma$-matrix interaction between the same two lines). Part of the $\Lambda$-particle self-energy $\Sigma_{2}$ is then found by calculating the two sets formed by first closing $\mathrm{N}_{1}-\mathrm{N}_{1}^{\prime}$ and $\mathrm{N}_{2}-\mathrm{N}_{2}^{\prime}$ with nucleon hole lines, and second the exchange diagrams formed by closing $\mathrm{N}_{1}-\mathrm{N}_{2}^{\prime}$ and $\mathrm{N}_{2}-\mathrm{N}_{1}^{\prime}$ with nucleon hole lines. The only other diagram with two distinct nucleon hole lines which cannot be included in these sets is shown in fig. $8 \mathrm{~b}$ (note that $k_{\mathrm{n}}=k_{\mathrm{n}}^{\prime}$ and hence the diagram only contains two distinct nucleon-hole lines). The self energy $\Sigma_{2}$ is then found by the sum of fig. $8 \mathrm{~b}$ and all diagrams arising from fig. $8 \mathrm{a}$ as indicated above. It is important to realize that the extended G-method treats these diagrams as Feynman diagrams and that the intermediate $\Lambda$-particle propagator is the unperturbed $G_{\Lambda}^{0}$. We note that the diagrams of which fig. $8 \mathrm{a}$ is representive have to include at least three $\Gamma$-matrix interactions. The possible diagrams containing two $\Gamma$-matrix interactions are shown in fig. 9. The contribution of fig. $9 a$ is excluded since it is not a proper self-energy and is included as an iteration of the first-order propagator. Fig. 9b which is the exchange closing of fig. 9a is invalid since it contains two consecu- 


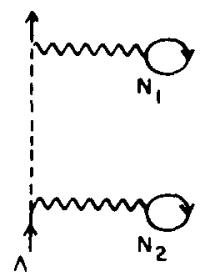

(a)

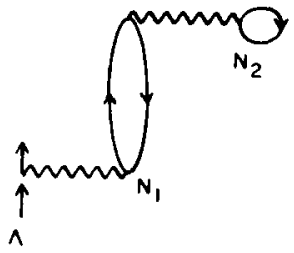

(c)

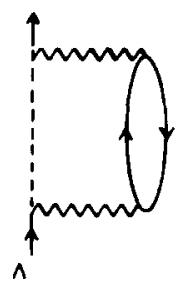

(b)

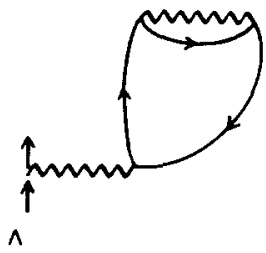

(d)

Fig. 9. Diagrams which can be neglected in evaluating the second-order cluster contribution to the $\Lambda$-particle self-energy, as explained in the text.

tive $\Gamma$-matrix interactions between the same two lines. Figs. $9 \mathrm{c}$ and $9 \mathrm{~d}$ will be included in fig. 1 for $\Sigma_{1}$, if the intermediate $G_{N}$ propagator is that found by a similar lowest-order G-calculation for NN pairs. This can again be represented crudely by an effective mass approximation for $G_{\mathrm{N}}$ but with a slightly lower value of $m_{N}^{*}$ than that found by the BG method, the difference arising from a similar difference to that already described in the $\Lambda \mathrm{N}$ case. To the extent that we are only considering the second-order calculation for $B_{\Lambda}$ this difference is unimportant, but in higher orders it would be significant.

The self-energy insertion of fig. $8 \mathrm{~b}$ is responsible for the so-called hole rearrangement effect and is readily evaluated. Dabrowski and Köhler [17] have evaluated it in the extended BG method where the diagram is treated as a Goldstone diagram. They have also considered two of the diagrams like fig. 8a, but this is inconsistent since they form part of an infinite set all of comparable magnitude. This set should be summed, and this could be done by methods similar to the Bethe-Faddeev techniques used by Bethe for nuclear matter.

In the extended G-formulation, it is now easy to calculate $B_{\Lambda}$ to second order. In lowest order we solved $p_{0}=\Sigma_{1}\left(0, p_{0}\right)=\Sigma_{1}\left(p_{0}\right)$ whose solution was given by $-B(1)$. To second order we must solve

$$
p_{\mathrm{o}}=\Sigma_{1}\left(p_{\mathrm{o}}\right)+g \Sigma_{2}\left(p_{\mathrm{o}}\right),
$$

where $g$ is a parameter introduced to keep track of the order of the terms and is finally set equal to unity. Let eq. (7.1) have solution 


$$
p_{\mathrm{o}}=-B_{\Lambda}^{(1)}-g B_{\Lambda}^{(2)}
$$

Then to $0(g)$ we may write

$$
-B_{\Lambda}^{(1)}-g B_{\Lambda}^{(2)}=\Sigma_{1}\left(-B_{\Lambda}^{(1)}\right)-\left.g B_{\Lambda}^{(2)} \frac{\mathrm{d} \Sigma_{1}}{\mathrm{~d} p_{\mathrm{o}}}\right|_{-B_{\Lambda}^{(1)}}+g \Sigma_{2}\left(-B_{\Lambda}^{(1)}\right)
$$

which correct to the order we are working has solution

$$
B_{\Lambda}=B_{\Lambda}^{(1)}-\frac{\Sigma_{2}{ }^{\left(-B_{\Lambda}^{(1)}\right)}}{1-\left.\frac{\mathrm{d} \Sigma_{1}}{\mathrm{~d} p_{\mathrm{o}}}\right|_{-B} ^{(1)}}
$$

It is important to notice to second order for $B_{\Lambda}$ the difference between the $\mathrm{BG}$ and $\mathrm{G}$-values for $B^{(1)}$ is now important.

To sum up, the extended G-technique, using Green-functions leads to a well-defined prescription for evaluating $B_{\Lambda}$ to any order (in the number of nucleon-hole lines in the proper self energy). Its beauty lies in the fact that the diagrams for the self energy can be treated as Feynman diagrams, having unique values independent of the rest of the diagram in which they are inserted. In these diagrams the internal $\Lambda$-particle propagators can be regarded as free propagators (as the exact prescription of Burkhardt [8] demands) and the self-consistency is guaranteed to the order to which the self-energy is calculated. In contrast the extended BG method which treats the diagrams as Goldstone diagrams loses these properties. The concept of off-the-energy-shell propagation has to be built in artificially in a self-consistent manner which is difficult. The results for any order have to be modified in passing to the next highest order, whereas in the extended Gmethod the values of the diagrams for any order are unchanged in passing to the next order; only the final equation for $B_{\Lambda}$ changes. Presumably the two methods both properly carried out to any order must only differ in higher-order terms. The point that we should like to make is that the Gmethod is much simpler in this case than the BG method which is fraught with pitfalls in building in the self-consistency requirements which are different in each order.

The general prescription outlined for the extended G-method is easily extended to higher orders, and is of course valid for all potentials, not just separable ones $\$$.

\section{SUMMARY}

Using an S-wave separable potential, we have been able to fit the lowenergy $\Lambda \mathrm{N}$ scattering data. The potential was chosen to include both attraction and repulsion. Using such a potential, one can solve exactly for the binding energy of a $\Lambda$-particle in nuclear matter by both the Bethe-Gold-

$\ddagger$ For a somewhat different treatment of the systematic inclusion of higher-order terms in pure nuclear matter, see ref. [18]. 
stone independent pair (BG) method and by a Green-function formalism due to Galitskii (G) where we kept just two-body clusters (ladder diagrams). The effect of the NN interactions was taken into account very crudely by replacing the nucleon mass by an effective mass. The BG method was shown to agree with the G-method in the limit of low-nuclear-matter density. For a realistic density of nuclear matter we showed that for $m_{\mathrm{N}}^{*}=0.65 m_{\mathrm{N}}$, corresponding to the self-consistent effective mass at the Fermi surface, the two methods differ by about $10 \%$, the G-method giving less binding. Furthermore, both methods are in essential agreement with the values found by other authors who consider the $S$-wave part of a local $\Lambda \mathrm{N}$ potential (see e.g. ref. [1]). For a value of $m_{\mathrm{N}}^{*}=m_{\mathrm{N}}$, however, the discrepancy between the two methods is much larger.

We then considered how the two approaches can be extended to higher orders, where the order of an approximation is reckoned to be the number of nucleon-hole lines in the $\Gamma$-matrix interaction diagrams for the proper self energy. We showed explicitly that for quite general potentials, the lowest order BG and G-methods give results which should be considered identical since they differ only by terms of higher order. The difference in the first-order results is important however if we wish to do a consistent second-order approximation for $B_{\Lambda}$. We showed an explicit procedure for extending the G-method to higher orders. The BG method can also be extended but the necessity of making it self-consistent, which is automatically built into the expanded G-method, makes it cumbersome and difficult. The extended G-method is guaranteed to give us results for $B_{\Lambda}$, which is the real chemical potential for the problem, correct to the order in which the self energy is calculated. The programme we gave for the extended Gmethod calculation of $B_{\Lambda}$ is valid for all $\Lambda \mathrm{N}$ potentials. The separable potential form is convenient for first-order solutions, and we showed that the closeness of the lowest-order BG and G-results indicates that the variousorder results for $B_{\Lambda}$ should converge quickly. In practice, this means that a second-order calculation using a realistic potential would give very precise results.

We stress again that the calculations with the separable potential do not give reliable results for $B_{\Lambda}$ but merely indicate the order of magnitude of the error incurred in using the BG approach naively when extended to second order without changing the self-consistency requirements from first order. There is no reason to suspect that using a more realistic potential would substantially alter these results.

Even in lowest order, there are several ways in which the separable potential model could be made more realistic. Firstly we would like to include higher-order partial waves than just $S$-waves. It is a trivial matter to extend the formalism to a separable potential of the form

$$
\left\langle\boldsymbol{k}|V| \boldsymbol{k}^{\prime}\right\rangle=\frac{\hbar^{2}}{2 \mu} \sum_{l=0}^{\infty} \lambda_{l} v_{l}(k) v_{l}\left(k^{\prime}\right) P_{l}\left(\hat{\boldsymbol{k}} \cdot \hat{\boldsymbol{k}}^{\prime}\right),
$$

but the difficulty now comes in choosing expressions for the functions $v_{l}$, and for the higher waves there is essentially no experimental data to quide us. 
A more serious problem is the use of the effective mass approximation for the $G_{\mathrm{N}}$ propagator. It is well known that this approximation is quite good for hole states with $k_{\mathrm{N}}<k_{\mathrm{F}}$, but is quite bad for particle states with $k_{\mathrm{N}}>k_{\mathrm{F}}$, and as we saw, our results are quite sensitive to the value of $m_{\mathrm{N}}^{*}$ used.

The potential we used was central and even if we use a more realistic local potential, we ought really to include the effects of a tensor piece, and of the coupling of the $\Lambda \mathrm{N}$ channel to the $\Sigma \mathrm{N}$ channel.

In their excellent review article Bodmer and Rote [4] indicate how to approach some of these problems within the extended BG formalism. This author is presently investigating the possibilities of obtaining a consistent second-order calculation of $B_{\Lambda}$ by the extended G-method.

The author would like to thank Professor J.D. Walecka for suggesting this problem and for many fruitful discussions during its progress.

\section{APPENDIX A}

Further comments on the BG and G-methods

In this appendix we wish to show explicitly the difference between the lowest-order results for the binding energy $B_{\Lambda}$ by the two methods, and show that the difference is in higher-order terms.

From eq. (6.4) is should be apparent that $B_{\Lambda}^{(\mathrm{G})}$ and $B_{\Lambda}^{(\mathrm{BG})}$ differ by terms involving at least three $\widetilde{\Gamma}$-matrices. To be more quantitative the lowest order iterate (in the number of $\widetilde{\Gamma}$-matrices) of eqs. (6.3) and (6.4) is

$$
\begin{aligned}
& B_{\Lambda}^{(\mathrm{G})}-B_{\Lambda}^{(\mathrm{BG})} \approx-4 B \Lambda_{\Lambda}^{(\mathrm{G})} \int_{0}^{k_{\mathrm{F}}} \frac{\mathrm{d}^{3} k}{(2 \pi)^{3}} \int \frac{\mathrm{d}^{3} k^{\prime}}{(2 \pi)^{3}}\left[\frac{2 \mu^{*} \tilde{\Gamma}\left(\boldsymbol{k}^{\prime}, \boldsymbol{q} ; \boldsymbol{P}_{(0)}\right) \theta\left(\mid \frac{\mu^{*}}{m_{\Lambda}} \boldsymbol{k}^{-} \boldsymbol{k},-\boldsymbol{k F}_{\mathrm{F}}\right)^{2}}{\boldsymbol{q}^{2}-\boldsymbol{k}^{2}+i \eta}\right] \\
& =-4 B \Lambda_{\Lambda}^{(\mathrm{G})} \int_{0}^{k} \frac{\mathrm{d}^{3} k}{(2 \pi)^{3}} \int \frac{\mathrm{d}^{3} k^{\prime}}{(2 \pi)^{3}}\left|\tilde{\chi}\left(\boldsymbol{k}^{\prime}, \boldsymbol{q} ; \boldsymbol{P}_{(0)}\right)-(2 \pi)^{3} \delta\left(\boldsymbol{k}^{\prime}-\boldsymbol{q}\right)\right|^{2},
\end{aligned}
$$

where the last equation follows from eq. (6.1). Thus

$$
B_{\Lambda}^{(\mathrm{G})}-B_{\Lambda}^{(\mathrm{BG})} \approx-4 B_{\Lambda}^{(\mathrm{G})} \int_{0}^{k \mathrm{~F}} \frac{\mathrm{d}^{3} k}{(2 \pi)^{3}} \int \mathrm{d}^{3} r \mid u_{\boldsymbol{P}_{(0)},\left.\boldsymbol{q}^{(r)}\right|^{2},}
$$

where

$$
{ }^{u} \boldsymbol{P}, \boldsymbol{k}(\boldsymbol{r})=\psi_{\boldsymbol{P}, \boldsymbol{k}}(\boldsymbol{r})-\mathrm{e}^{i \boldsymbol{k} \cdot \boldsymbol{r}}
$$

is the BG deficit wave function.

Eq. (A.2) can be written slightly differently if we realize that the BG wave function for a realistic hard-core potential has a negligible dependence on the total momentum $\boldsymbol{P}$ and can be evaluated at $\boldsymbol{P}=\boldsymbol{0}$ for the pur- 
poses of eq. (A.2). If we define $\left\langle u_{\Lambda \mathrm{N}}^{2}\right\rangle$ to be the value of $\left|u_{\boldsymbol{P}(0), q}\right|^{2}$ evaluated at $P_{(0)}=0$ and averaged over the Fermi sea, and realising that

$$
4 \int_{0}^{k} \frac{\mathrm{d}^{3} k}{(2 \pi)^{3}}=\frac{A}{\Omega}=\rho,
$$

we can write eq. (A.2) as

$$
\begin{aligned}
B_{\Lambda}^{(\mathrm{G})}-B_{\Lambda}^{(\mathrm{BG})} & \approx-B_{\Lambda}^{(\mathrm{G})} \rho \int \mathrm{d}^{3} r\left\langle u_{\Lambda \mathrm{N}}^{2}(r)\right\rangle \\
& =-\omega_{\Lambda \mathrm{N}} B_{\Lambda}^{(\mathrm{G})} .
\end{aligned}
$$

In sect. 7 , we indicated that $\omega_{\Lambda N}$ is a typical expansion parameter in the extended BG formalism, and hence from eq. (A.3) we see explicitly that our first-order results by the two methods differ only in higher-order terms.

\section{APPENDIX B}

Galitskii formalism for a pure hard-core $\Lambda \mathrm{N}$ interaction

We now consider the case of a $\Lambda \mathrm{N}$ local hard-core interaction,

$$
V(\boldsymbol{r})= \begin{cases}\infty, & r<a \\ 0, & r>a\end{cases}
$$

In this case, the Fourier transform of the potential is undefined. The gist of the Galitskii method is to realise that even if $V$ blows up, the scattering amplitude remains finite. For a local potential, eq. (3.5) becomes

$$
\begin{aligned}
\chi\left(\boldsymbol{p}, \boldsymbol{p}^{\prime} ; P\right)=(2 \pi)^{3} \delta\left(\boldsymbol{p}^{\prime}-\boldsymbol{p}\right)+\frac{\theta\left(\left|\frac{1}{2}(1-\alpha) \boldsymbol{P}-\boldsymbol{p}\right|-k_{\mathbf{F}}\right)}{\left(\epsilon-\boldsymbol{p}^{2}+i \eta\right)} \\
\quad \times \int \frac{\mathrm{d}^{3} q}{(2 \pi)^{3}} u(\boldsymbol{q}) \times\left(\boldsymbol{p}-\boldsymbol{q}, \boldsymbol{p}^{\prime} ; P\right) .
\end{aligned}
$$

We define a function $x_{0}$ by:

$\chi_{\mathrm{o}}\left(p, \boldsymbol{p}^{\prime} ; P\right)=(2 \pi)^{3} \delta\left(\boldsymbol{p}^{\prime}-\boldsymbol{p}\right)+\frac{1}{\left(\epsilon-\boldsymbol{p}^{2}+i \eta\right)} \int \frac{\mathrm{d}^{3} q}{(2 \pi)^{3}} u(\boldsymbol{q}) \mathrm{x}_{\mathrm{o}}\left(\boldsymbol{p}-\boldsymbol{q}, \boldsymbol{p}^{\prime} ; P\right)$.

If there are no bound states of the $V_{\Lambda N}$ potential, then $\psi_{\boldsymbol{k}}(\boldsymbol{p})$, the exact c.m. two-body scattering wave functions, form a complete set, and the expansion

is easily verified.

$$
\chi_{\mathrm{o}}\left(\boldsymbol{p}, \boldsymbol{p}^{\prime} ; P\right)=\left(\epsilon-\boldsymbol{p}^{\prime 2}+i \eta\right) \int \frac{\mathrm{d}^{3} k}{(2 \pi)^{3}} \frac{\psi_{\boldsymbol{k}}(\boldsymbol{p}) \psi_{\boldsymbol{k}}^{*}\left(\boldsymbol{p}^{\prime}\right)}{\left(\epsilon-\boldsymbol{k}^{2}+i \eta\right)}
$$

The Lippmann-Schwinger equation is used to substitute for $\psi_{\boldsymbol{k}}^{*}\left(\boldsymbol{p}^{\prime}\right)$ : 
where

$$
\psi_{\boldsymbol{k}}^{*}\left(\boldsymbol{p}^{\prime}\right)=(2 \pi)^{3} \delta\left(\boldsymbol{p}^{\prime}-\boldsymbol{k}\right)+\frac{\widetilde{f}^{*}\left(\boldsymbol{p}^{\prime}, \boldsymbol{k}\right)}{\left(\boldsymbol{k}^{2}-\boldsymbol{p}^{2}-i \eta\right)}
$$

$$
\tilde{f}(\boldsymbol{p}, k)=\int \frac{\mathrm{d}^{3} q}{(2 \pi)^{3}} u(\boldsymbol{q}) \psi_{\boldsymbol{k}}(\boldsymbol{p}-\boldsymbol{q}) .
$$

Defining $\Gamma_{0}$ in a similar way to $\Gamma$,

$$
2 \mu^{*} \Gamma_{\mathrm{o}}\left(\boldsymbol{p}, \boldsymbol{p}^{\prime} ; P\right)=\int \frac{\mathrm{d}^{3} q}{(2 \pi)^{3}} u(\boldsymbol{q}) \mathrm{X}_{\mathrm{o}}\left(\boldsymbol{p}-\boldsymbol{q}, \boldsymbol{p}^{\prime} ; P\right),
$$

we find

$$
\begin{aligned}
2 \mu^{*} \Gamma_{\mathrm{o}}\left(\boldsymbol{p}, \boldsymbol{p}^{\prime} ; P\right)=\tilde{f}\left(\boldsymbol{p}, \boldsymbol{p}^{\prime}\right)+\int \frac{\mathrm{d}^{3} k}{(2 \pi)^{3}} \tilde{f}(\boldsymbol{p}, \boldsymbol{k}) \tilde{f}^{*}\left(\boldsymbol{p}^{\prime}, \boldsymbol{k}\right) \\
\times\left[\frac{1}{\epsilon-\boldsymbol{k}^{2}+i \eta}+\frac{1}{\boldsymbol{k}^{2}-\boldsymbol{p}^{2}-i \eta}\right] .
\end{aligned}
$$

Using eqs. (B.1), (B.2) and (B.4),

$$
\begin{aligned}
\Gamma\left(\boldsymbol{p}, \boldsymbol{p}^{\prime} ; P\right)=\Gamma_{\mathrm{o}}\left(\boldsymbol{p}, \boldsymbol{p}^{\prime} ; P\right)-\int \frac{\mathrm{d}^{3} k^{\prime}}{(2 \pi)^{3}} \Gamma_{\mathrm{o}}\left(\boldsymbol{p}, \boldsymbol{k}^{\prime} ; P\right) & \frac{\theta\left(k_{\mathrm{F}}-\left|\frac{1}{2}(1-\alpha) \boldsymbol{P}-\boldsymbol{k}^{\prime}\right|\right)}{\epsilon-\boldsymbol{k}^{2}+i \eta} \\
& \times 2 \mu^{*} \Gamma\left(\boldsymbol{k}^{\prime}, \boldsymbol{p}^{\prime} ; P\right)
\end{aligned}
$$

Corect to second order in $\tilde{f}$, we may replace $\Gamma$ by $\Gamma_{\mathrm{O}}$ in the integral of eq. (B.6). In the limit $\delta_{\mathrm{O}}=-k a \rightarrow 0$,

$$
\tilde{f}\left(\boldsymbol{k}, \boldsymbol{k}^{\prime}\right)=-4 \pi f\left(\boldsymbol{k}, \boldsymbol{k}^{\prime}\right) \rightarrow 4 \pi\left(a-i k a^{2}\right)+\mathrm{O}\left(k^{2} a^{3}\right),
$$

where $a$ is the scattering length or radius of the hard core.

In all that follows we now work to $\mathrm{O}\left(a^{2}\right)$. From eq. (B.5):

$$
2 \mu^{*} \Gamma_{\mathrm{o}}(\boldsymbol{q}, \boldsymbol{q} ; P)=4 \pi a+16 \pi^{2} a^{2} \int \frac{\mathrm{d}^{3} k}{(2 \pi)^{3}}\left[\frac{1}{\epsilon-\boldsymbol{k}^{2}+i \eta}+\frac{\mathrm{P} \cdot \mathrm{V} .}{\boldsymbol{k}^{2}-\boldsymbol{q}^{2}}\right],
$$

and hence from eq. (B.6)

$2 \mu^{*} \Gamma(\boldsymbol{q}, \boldsymbol{q} ; P)=4 \pi a+16 \pi^{2} a^{2} \int \frac{\mathrm{d}^{3} k^{\prime}}{(2 \pi)^{3}}\left[\frac{\theta\left(\left|\frac{1}{2}(1-\alpha) \boldsymbol{P}-\boldsymbol{k}^{\prime}\right|-k_{\mathrm{F}}\right)}{\epsilon-\boldsymbol{k}^{2}+i \eta}+\frac{\mathrm{P} \cdot \mathrm{V} .}{\boldsymbol{k}^{2}-\boldsymbol{q}^{2}}\right]$.

Hence $\Sigma$ can be found from eq. (3.8).

The excitation spectrum is given by the poles of $G_{\Lambda}$, or the solutions to $p_{\mathrm{O}}-\boldsymbol{p}^{2} / 2 m_{\Lambda}-\Sigma\left(\boldsymbol{p}, p_{\mathrm{O}}\right)=0$. Since this implies $p_{\mathrm{O}}=\boldsymbol{p}^{2 / 2} m_{\Lambda}+\mathrm{O}\left(k_{\mathrm{F}} a\right)$, it is consistent to $\mathrm{O}\left(k_{\mathrm{F}} a\right)^{2}$ to replace $p_{\mathrm{O}}$ by $p^{2 / 2} m_{\Lambda}$ in eq. (B.7) to give 


$$
\begin{aligned}
p_{\mathrm{O}}=\frac{p^{2}}{2 m_{\Lambda}}+\frac{\hbar^{2} k_{\mathrm{F}}^{2}}{2 \mu^{*}}\left[\frac{8 k}{3 \pi}+\frac{64 \pi^{2} a^{2}}{k_{\mathrm{F}}^{2}} \int \frac{\mathrm{d}^{3} k}{(2 \pi)^{3}} \int \frac{\mathrm{d}^{3} k^{\prime}}{(2 \pi)^{3}} \theta\left(k_{\mathrm{F}}-k\right)\right. \\
\left.\quad \times\left\{\frac{\mathrm{P} \cdot \mathrm{V} .}{\boldsymbol{k}^{\prime 2}-\boldsymbol{q}^{2}}+\frac{\left.\theta\left(\left|\frac{1}{2}(1-\alpha) \boldsymbol{P}-\boldsymbol{k}^{\prime}\right|-k \mathrm{~F}\right)\right\}}{\boldsymbol{q}^{2}-\boldsymbol{k}^{2}+i \eta}\right\}\right] .
\end{aligned}
$$

For $p=0$, evaluation of eq. (B.8) gives the same result for the groundstate energy as found by Walecka [1] who used the Bethe-Goldstone method.

Eq. (B.8) can be written as

$$
p_{\mathrm{o}} \equiv \varepsilon(p)=\frac{p^{2}}{2 m_{\Lambda}}+F(p)
$$

and making a Taylor expansion of $F(p)$ around $p=0$,

$$
\varepsilon(p)-\varepsilon(0) \equiv \frac{p^{2}}{2 m_{\Lambda}^{*}}=\frac{p^{2}}{2 m_{\Lambda}}+\frac{p^{2}}{2} F^{\prime \prime}(0),
$$

since $F^{\prime}(0)=0$, where this equation defines $m_{\Lambda}^{*}$, we find

$$
\frac{1}{m_{\Lambda}^{*}}=\frac{1}{m_{\Lambda}}+\frac{8\left(a{ }^{2}\right)^{2}}{3 \pi^{2} \mu^{*}}\left[\frac{(1-\alpha)^{3}}{4 \alpha^{2}} \ln \left(\frac{1+\alpha}{1-\alpha}\right)-\frac{(1-\alpha)}{2 \alpha}\right],
$$

where

$$
\alpha=\frac{m_{\Lambda}-m_{\mathrm{N}}^{*}}{m_{\Lambda}+m_{\mathrm{N}}^{*}}
$$

We see that $m_{\Lambda}^{*}>m_{\Lambda}$ as expected.

Evaluation of eq. (B.10) at $k_{\mathrm{F}}=1.35 \mathrm{fm}^{-1}, a=0.4 \mathrm{fm}, m_{\mathrm{N}}^{*}=0.65 m_{\mathrm{N}}$ yields $m_{\Lambda}^{*} / m_{\Lambda}=1.15$, which is really not negligible.

From eq. (B.8) we can also find the damping term $\gamma(p)$ of the quasi-particle spectrum. Writing the solution to eq. (B.8) in the form $p_{\mathrm{O}}=\varepsilon(p)+i \gamma(p)$, we find

$$
\gamma(p)=-\frac{4}{15 \pi}\left(\frac{\hbar^{2} k_{\mathrm{F}}^{2}}{m}\right)\left(a k_{\mathrm{F}}\right)^{2} \times\left\{\begin{array}{cc}
2 x^{4}, & 0<x<1 \\
\left(5 x-\frac{3}{x}\right), & x>1,
\end{array}\right.
$$

where $x=p / k_{\mathrm{F}}$, and $\gamma(p)$ has been evaluated, for simplicity, with $m_{\mathrm{N}}^{*}=m_{\Lambda}=m$.

\section{REFERENCES}

[1] J.D.Walecka, Nuovo Cimento 16 (1960) 342.

[2] D. H. Davis, R.Levi-Setti, M.Raymund, O.Skjeggestad, G. Tomasini, J.Lemonne, P.Renard and J.Sacton, Phys. Rev. Letters 9 (1962) 464. 
[3] R.H. Dalitz, Hypernuclear interactions, Proc. of the topical conf. on the use of elementary particles in nuclear structure studies (Brussels, September 1965).

[4] A.R. Bodmer and D. M. Rote, to be published.

[5] H.A. Bethe and J.Goldstone, Proc. Roy. Soc. A238 (1957) 551.

[6] K.A. Brueckner and J. L.Gammel, Phys. Rev. 109 (1958) 1023.

[7] L.C.Gomes, J.D.Walecka and V.F.Weisskopf, Ann. of Phys. 3 (1958) 241.

[8] T.W. Burkhardt, Nucl. Phys. A120 (1968) 434.

[9] V.M.Galitskii, JETP (Sov. Phys.) 7 (1958) 104.

[10] V.J.Emery and A. M.Sessler, Phys. Rev. 119 (1960) 248;

A. Bohr, B.R. Mottelson and D. Pines, Phys. Rev. 110 (1958) 936.

[11] H. Sugawara and F. Von Hippel, Phys. Rev. 172 (1968) 1764.

[12] R.C.Herndon and Y.C.Tang, Phys. Rev. 159 (1967) 853.

[13] G.Alexander, O.Benary, U. Karshon, A. Shapira, G. Yekutieli, R. Engelmann, H. Filthuth and B.Schiby, Phys. Letters 19 (1966) 715.

[14] G. Alexander, U. Karshon, A.Shapira, G. Yekutieli, R. Engelmann, H. Filthuth and W. Lughofer, Phys. Rev. 173 (1968) 1452.

[15] F. Tabakin, Phys. Rev. 174 (1968) 1208.

[16] H. A. Bethe, Phys. Rev. B138 (1965) 804;

R. Rajaraman and H.A. Bethe, Revs. Mod. Phys. 39 (1967) 745.

[17] J.Dabrowski and H.S. Köhler, Phys. Rev. B136 (1964) 162.

[18] B. Brandow, Phys. Rev. 152 (1966) 863. 